This is in the post peer review accepted manuscript of:

G. C. Montanari, P. Seri, M. Karttunen, M. Paajanen, K. Lahti and I. Rytöluoto, "Nanocomposite polypropylene for DC cables and capacitors: A new European project," 2017 International Symposium on Electrical Insulating Materials (ISEIM), Toyohashi, 2017, pp. 433-436. doi: 10.23919/ISEIM.2017.8088777

The published version is available online at: https://doi.org/10.23919/ISEIM.2017.8088777

(C) 2017 IEEE. Personal use of this material is permitted. Permission from IEEE must be obtained for all other uses, in any current or future media, including reprinting/republishing this material for advertising or promotional purposes, creating new collective works, for resale or redistribution to servers or lists, or reuse of any copyrighted component of this work in other works 


\title{
Nanocomposite Polypropylene For DC Cables And Capacitors: A New European Project.
}

\author{
Gian Carlo Montanari ${ }^{1}$, Paolo Seri ${ }^{1}$, Mikko Karttunen ${ }^{2}$, Mika Paajanen ${ }^{2}$, Kari Lahti ${ }^{3}$ and Ilkka Rytöluoto ${ }^{3}$ \\ ${ }^{1}$ Electrical, Electronic and Information Engineering “Guglielmo Marconi” Dept. (DEI), University of Bologna, Bologna (BO), Italy \\ ${ }^{2}$ VTT Technical Research Centre of Finland Ltd., Tampere, Finland \\ ${ }^{3}$ Tampere University of Technology, Department of Electrical Engineering, Tampere, Finland \\ E-mail: giancarlo.montanari@unibo.it
}

\begin{abstract}
This paper presents the scientific background of a new European project, GRIDABLE, which was launched at the beginning of 2017 and has to deliver results in manufacturing and characterization of LV-MV capacitors and MV-HV cables for DC application. The innovation is in the development of nanostructured materials based on polypropylene and silica, and the relevant capacitor and cable manufacturing procedures. The initial results regarding the electrical properties of $\mathrm{PP}_{-} \mathrm{SiO}_{2}$ materials, which have brought to the proposal of this project, are presented in this paper, focusing on breakdown strength and space charge measurements performed on nanofilled PP films for capacitors.
\end{abstract}

Keywords-HVDC; space charge; nanocomposite; DC cable; Capacitor; $\mathrm{SiO}_{2}$

\section{INTRODUCTION}

A new European project, that is, GRIDABLE (Polymer Nanocomposite Insulation Material Enabling Integration of Renewables and DC Storage Technologies in the AC Energy Grid) was launched recently under the HORIZON 2020 framework funded by the European Community. Various partners, from universities and research institutions to cable and capacitor manufactures, are involved. The project aim is to develop a new generation of nanostructured polymeric materials suitable for DC cables and capacitors. In addition, the project aims in its last stage to launch, on a pre-production scale, industrial prototypes of DC power capacitors and cables using industrial machinery throughout the production chain.

The base material chosen for this project is thermoplastic, i.e. polypropylene (PP); having excellent electrical and thermal properties for energy applications, and good potentiality regarding mechanical properties, besides being completely recyclable. The choice to add nanofillers, specifically $\mathrm{SiO}_{2}$ with and without functionalization, fits to the objective to improve the electrical and thermal properties and reach higher design field and temperature for the same design life and reliability of the present DC technology, that is, XLPE (cross-linked polyethylene) for cables and PP for capacitors.

Preliminary work, presented in this paper, indicates that such objectives can be reached, upon proper selection of the base PP and surface treatment of the nanofillers. The paper describes the criteria for material selection, the preliminary test data, and discusses the potentiality of the project in comparison with the present materials and technologies used in DC cables and capacitors, with particular emphasis to capacitors development. Breakdown strength and space charge test results are shown which may support the developments which are the aim of the project.

\section{THE PROJECT}

The aim of GRIDABLE is to develop $\mathrm{PP} / \mathrm{SiO}_{2}$ nanocomposites with proper nanoparticle functionalization and dispersion, demonstrating the improvements this novel material can bring to the state of the art of HVDC cables and DC capacitors insulation. Under high DC electric fields, XLPE insulation is currently the typical solution proposed by the cable industry. Such material may experience reliability issues related to the formation of space charge, which can happen through slow charge injection and transport, or through fast charge pulses [1],[2]. In addition, XLPE is not recyclable and its processing is quite energy intensive and complex.

Regarding space charge suppression, or reduction, it has been shown already that space charge injection can be minimized by controlling polymer morphology and crystallinity, and accumulation can be minimized working on charge mobility adding nanoparticles able to create low-energy trapping sites at the nanoparticle surface and on the interaction zones between the nanoparticle and the polymer matrix [3]-[5].

Different space charge accumulation choosing a $\mathrm{PP} / \mathrm{SiO}_{2}$ nanocomposite over XLPE could contribute to solve major issues coming from long term or single polarity switching event. The production speed is also expected to be higher, and the total energy consumption of the $\mathrm{PP} / \mathrm{SiO}_{2}$ processing to be significantly lower, thanks to the reduction in the melt viscosity and the absence of the curing/degassing steps when using $\mathrm{PP} / \mathrm{SiO}_{2}$. Moreover, recyclable $\mathrm{PP} / \mathrm{SiO}_{2}$ can allow having thermoplastic material with longer life cycle, which is in agreement also with IEC recommendations. The electrical characterization and long term reliability assessments for this material will be defined through simultaneous accelerated voltage and temperature conditioning, statistical models for life and large-area multi-breakdown analysis. Once this novel HVDC insulation material have been extensively tested and proved suitable for commercial production, the accumulated processing and material know-how can be utilized for preproduction scale composite compounding and extrusion for capacitor films and HVDC model cables, finally validating their quality according to the industrial standard.

Thus, the GRIDABLE project is not only focused on the applied research for a novel material, but rather in developing a complete industrial solution. 


\section{SPACE CHARGE AND AGING}

The accumulation of space charge in electrical insulation has been recognized to be a major aging factor in DC insulation [3][5]. As an example, Figure 1 shows how a small amount of accumulated space charge can distort the laplacian electric field. This will in turn influence the degradation rate and life expectancy of the insulating material. Due to the power law which describes the aging rate under electrical stress, even a moderate increase of electrical field inside the insulation can significantly accelerate the local degradation mechanism driven by electrical stress. Indeed, the relationship between aging rate, $K$, the electrical stress, $E$, and the so-called voltage endurance coefficient, $n$, can be described by the inverse power life model (IPM), defined by eq.1 [6][7]:

$$
K(E)=K_{D}\left(\frac{E}{E_{D}}\right)^{n}
$$

where $E_{D}$ is design field and $K_{D}$ the corresponding aging rate constant. If, e.g., the electric field inside insulation locally becomes $20 \%$ higher than ED, and considering a life of 30 years at failure probability $1 \%$ for a design field of $40 \mathrm{kV} / \mathrm{mm}$, and $\mathrm{n}=15$, the increase of electric field will bring to life reduction of $90 \%$, that is from 30 to 3 years.

Hence, accumulation of space charge can be the main accelerated degradation process in DC insulation. If, in addition, voltage polarity inversions phenomena are present (as it may happen in transmission assets), the accumulation of homocharge (that is, charge having the same sign as that of the near electrode) can bring to further abnormal field distribution, causing significant field enhancement at the interface insulation-electrode, which can affect apparatus reliability and life [8].

Any material which has to be used as DC insulation, therefore, has to be characterized to be space charge free at the operating field and temperature, and nanostructuration can help increasing field and/or temperature above which space charge accumulation becomes large enough to affect field profile and/or accelerate local degradation mechanisms [6],[9].

\section{TESTING PROCEDURES}

\section{A. Space charge measurements}

Space charge measurements were carried out by the Pulsed Electro-Acoustic (PEA) method. In the PEA method, a specimen is subject to the superposition of a DC voltage U0 and a pulsed voltage $U p(t)$. Charged particles injected by the DC field interact with the bulk material due to the pulsed voltage, producing an acoustic pressure wave that is transduced into a voltage signal by a piezoelectric sensor. The trend of this signal in time is then related to the charge distribution in space by known parameters, such as the sound velocity in the specimen, its thickness, and the applied electric field. Besides information on space charge profiles (i.e. charge density vs. distance) and their evolution as a function of the electric field, quantities were extracted from space charge profiles detected by PEA, such as threshold for space charge accumulation. This is a fundamental parameter for the characterization of insulating material for DC applications, because, as mentioned above, the presence of
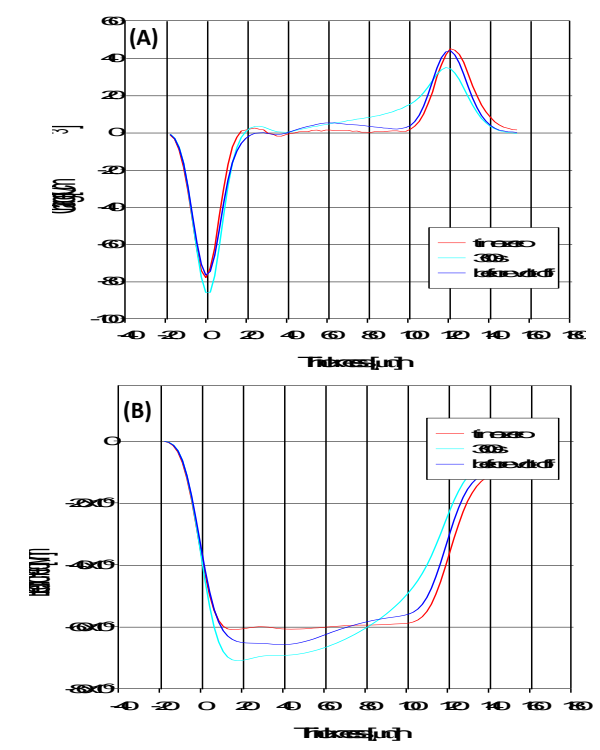

Figure 1 - Space charge (A) and electric field profile (B) measured at the beginning, during and at the end of polarization with (laplacian) field of 60 $\mathrm{kV} / \mathrm{mm}$. The maximum field bulk material is subject to is about $70 \mathrm{kV} / \mathrm{mm}$, after $300 \mathrm{~s}$.

space charge in the insulation can modify substantially the electric field distribution and, hence, the insulation life [10][12]. The space charge measurements were performed on thermally pre-treated specimens ( 72 hours at $60^{\circ} \mathrm{C}$ in vacuum). This is done to remove traces of water diffused into the material that can deeply influence the final results [13]. Measurements during 3-hour polarization (while DC voltage is applied) and depolarization (while DC voltage is absent) times are carried out in this work. Tests are done at $30^{\circ} \mathrm{C}$, in controlled environment, with fields ranging from 7.6 to $38.5 \mathrm{kV} / \mathrm{mm}$, in order to unravel the low field and high field behavior of space charge and, in particular, find out the threshold for space charge accumulation. The threshold field is obtained by identifying a bend from the linear ohmic relationship between the stored charge density at a specific field and depolarization time, $q(E, t)$, derived from the space charge profile measurements as follows:

$$
q(E, t)=\frac{1}{L} \int_{0}^{L}\left|q_{p}(E, x, t)\right| d x
$$

where 0 and $L$ denote the electrodes positions and $q_{p}(E, x, t)$ is the space charge profile for a given poling field $E$. Induced or capacitive charges at the electrodes are not taken into account. Calculation is made $2 \mathrm{~s}$ after switching off the voltage.

\section{B. DC multi-breakdown measurements}

Large-area DC multi-breakdown measurements were performed as described in [14]. In order to realize self-healing breakdown measurement, two $90 \mathrm{~mm}$ wide sheets of commercial Zn-Al-metallized BOPP film were used as electrode films, above and under the specimen film, thus forming a test capacitor with an active area of about $81 \mathrm{~cm}^{2}$. Electrical contacts with the metallized films were made by means of aluminum clamp electrodes at the both ends of the bottom plate. The breakdown measurements were performed in transformer oil, 
according to with the IEC-60243, in order to mitigate surface flashovers and other non-breakdown events occurring at the specimen and electrode film edges. During each discharge event in the test capacitor, discharge current, test capacitor voltage and time-signature of the event were recorded by a high-resolution oscilloscope. This allowed precise determination of breakdown voltage, voltage drop, peak current, discharge energy and various pulse time parameters, for each discharge event. A video was recorded from the top of the test capacitor unit for the whole duration of the breakdown measurement, thus allowing detailed chronological analysis of the breakdown progression after the measurement. The breakdown fields were calculated event-byevent by measuring the average thickness around each discharge spot by means of the video recording and the specimen film thickness map in MATLAB. Albeit more laborious, this procedure minimizes the error in the breakdown field calculation due to film thickness deviation [14]. Prior to the statistical analysis of the breakdown results, a data qualification procedure based on the discharge energy and breakdown voltage characteristics of the self-healing breakdown process was performed in MATLAB to exclude possible non-breakdown events from the measurement data (such as partial discharges and consecutive breakdowns occurring close to or at previous breakdown sites). A detailed description of the procedure is omitted here and can be found elsewhere [14]. Finally, for the statistical analysis of the qualified breakdown data sets, the 2parameter (2-p) Weibull distribution was used:

$$
F\left(V_{S}\right)=1-\exp \left[-\left(\frac{V_{S}}{\alpha}\right)^{\beta}\right]
$$

where $V_{S}$ is breakdown voltage (or field), $\alpha$ is the scale parameter, corresponding to failure probability $63.2 \%$, and $\beta$ is the shape parameter, related Weibull plot slope and, thus, to data dispersion. Maximum-likelihood estimation (MLE) and nonlinear regression (NLR) methods were used for the Weibull parameter estimation and the analysis was performed by Weibull++ and MATLAB software. One-sided and doublesided $90 \%$ confidence bounds were calculated for the Weibull parameters and breakdown percentiles by the Fisher Matrix (FM) method.

\section{MATERIAL SPECIFICATIONS AND COMPOUNDING}

The nanocompounds selected and characterized in this work include a capacitor-grade isotactic polypropylene homopolymer matrix, and 1.0-4.5 wt- $\%$ of hexamethyldisilazane (HMDS)-treated hydrophobic fumed silica nanoparticles. An antioxidant package consisting of a main process stabilizer and a co-stabilizer was added to each compound to prevent thermo-oxidative degradation of the PPmatrix during melt-processing. The raw materials were premixed, dried for $1.5 \mathrm{~h}$ in oven $\left(70^{\circ} \mathrm{C}\right)$ and for $0.5 \mathrm{~h}$ in vacuum, and thereafter compounded by a co-rotating twin-screw extruder. Continuous nitrogen gas purge and a melt filter were utilized. The compounded strands were cooled, pelletized and dried prior to film manufacturing. For laboratory-scale specimen manufacturing, the dried compounds were first extruded into $\sim 700 \mu \mathrm{m}$ cast films and stretched biaxially thereafter, resulting in films $\sim 15-20 \mu \mathrm{m}$ in thickness. Pilotscale films were manufactured at a Brückner pilot film line consisting of a single screw extruder with a flat die, a casting station, machine- and transverse directional orientation (MDO and TDO) units, and a winder. The selected compounds were melted in the extruder and cast on a chill roll which was set at a temperature of $62^{\circ} \mathrm{C}$. The film was then reheated and stretched in the MDO unit followed by reheating and stretching in the TDO unit. Biaxially stretched film thickness was in the range of $15-18 \mu \mathrm{m}$.

\section{PRELIMINARY EXPERIMENTAL RESULTS AND DISCUSSION}

\section{A. Space charge accumulation}

Figure 2A shows an example of space charge profiles obtained by PEA on specimens of the base and nanostructured materials. As can be seen, the difference is minimal. This is confirmed by the space charge threshold characteristics, showing that the threshold for space charge accumulation at $20^{\circ} \mathrm{C}$ is between 15 and $20 \mathrm{kV} / \mathrm{mm}$ for all tested materials. This value is in line with that found on best-grade XLPE in a previous EU-funded research project, that is HVDC.
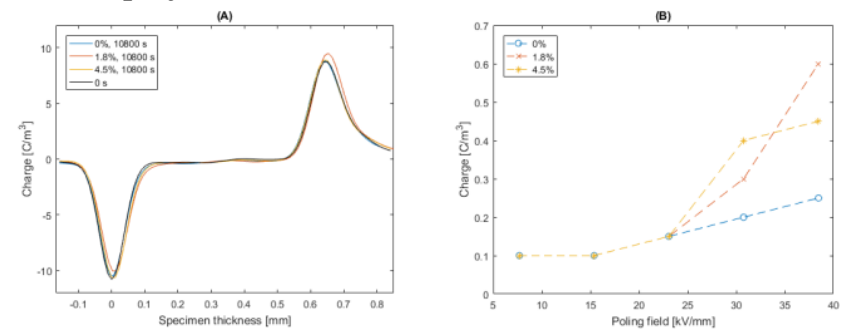

Figure 2 - Space charge profiles at $38 \mathrm{kV} / \mathrm{mm}$ (A) and space charge threshold characteristics (B) for PP pure and filled $1.8 \mathrm{wt} \%$ and $4.5 \mathrm{wt} \%$.

Such results look a promising basis to work on the improvement on nanofiller compatibilization and its dispersion in the base $\mathrm{PP}$, as well as to look at improved technological processes which can increase the space charge accumulation threshold and the rate of growth of charge amplitude as function of applied field.

\section{B LARGE-AREA DIELECTRIC BREAKDOWN PERFORMANCE}

The large-area multi-breakdown distributions of the 1.0-4.5 wt$\%$ laboratory-scale silica-BOPP nanocomposite films and an unfilled BOPP reference films are summarized by the Weibull plots of Figure 3A. Compared to the unfilled reference BOPP, it is shown that increasing silica content reduces the DC breakdown performance in the $>5 \%$ breakdown probability region, but it also leads to an increase of the shape parameter, that is to a decrease of the breakdown distribution dispersion [15]. Interestingly, the Weibull graphs of the $1.0 \mathrm{wt}-\%$ silicaBOPP and the unfilled BOPP intersect in the 5-10\% breakdown probability range, with the nanostructured BOPP showing higher electric strength in the low probability region. This is promising for the breakdown statistics of film capacitors, for which the design electric field is limited by the weakest points in the polymer film. Indeed, both the increase of the voltage breakdown distribution homogeneity and the improvement in the low-probability breakdown behavior may be considered as more significant factors for capacitor design 
than the breakdown behavior in the higher $(>10 \%)$ breakdown probability region.
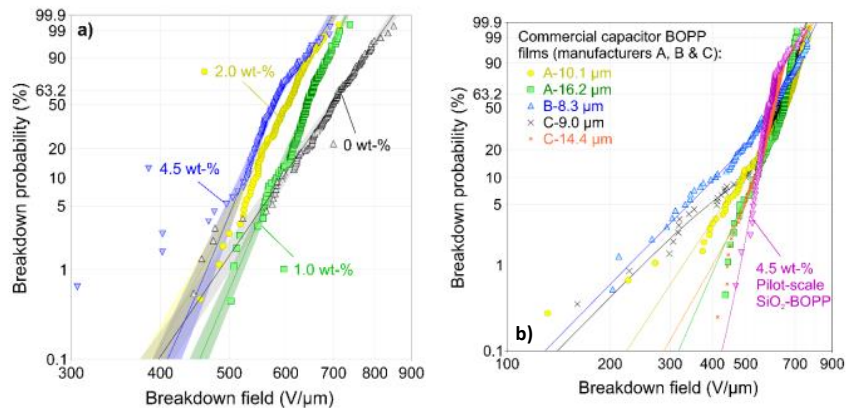

Figure 3 - Large-area multi-breakdown Weibull distributions of the studied nanocompounds at laboratory-scale, with 1.0 to $4.5 \mathrm{wt}-\%$ silica-BOPP, and unfilled reference $(\mathrm{A})$ and various commercial capacitor-grade BOPP films (manufacturers A, B \& $\mathrm{C}$ ) in the 8-16 $\mu \mathrm{m}$ thickness range in comparison with the $4.5 \mathrm{wt}-\%$ pilot-scale silica-BOPP nanocomposite film (B). The solid lines and shaded areas correspond to Weibull plots and their $90 \%$ confidence bounds, respectively.

Figure 3B shows the improved low-probability breakdown performance of $4.5 \mathrm{wt}-\%$ silica-BOPP pilot-scale film, in comparison with various commercial capacitor-grade BOPP films. As can be seen, the shape parameter of the Weibull distribution for the nanostructured material is significantly higher than those of the BOPP films, and again there is a crossing at low breakdown probability (this time about $10 \%$ ), which confirms the results of Figure 3A.

\section{CONCLUSIONS}

The addition of $\mathrm{SiO}_{2}$ nanoparticles to $\mathrm{PP}$ has revealed interesting modifications of electrical properties that may affect positively HVDC cables and film capacitors design and performance on short term and long term basis. The good behavior of DC breakdown voltage and the extent of the space charge accumulation field might open interesting perspectives for the future of the GRIDABLE project. Further, long term, investigations are needed to understand the contribution of nanofillers to the modification of electrical properties, such as measurements of charging-discharging currents and space charges at various temperatures, dielectric spectroscopy, life tests with voltage polarity inversion, but the results here reported seem to indicate that the GRIDABLE project may have an important impact on insulation technology in the near future.

\section{ACKNOWLEDGMENTS}

This project has received funding from the European Union's Horizon 2020 research and innovation programme under grant agreement No 720858.

\section{REFERENCES}

[1] D. Fabiani, G. C. Montanari and L. A. Dissado, "Space charge accumulation due to ultra-fast charge packets in XLPE insulated cables: the effect of temperature and field", IEEE Inter. Conf. Properties Applications Dielectr. Materials (ICPADM), pp. 337-340, 2009.

[2] S. Delpino, D. Fabiani, G. C. Montanari, L. A. Dissado, C. Laurent and G. Teyssedre, "Fast charge packet dynamics in XLPE insulated cable models", IEEE Conf. Electr. Insul. Dielectr. Phenomena (CEIDP), pp. $421-424,2007$

[3] Tanaka, T; Bulinski, A; Castellon, J; at al., "Dielectric properties of $\mathrm{XLPE} / \mathrm{SiO} 2$ nanocomposites based on CIGRE WG D1.24 cooperative test results", IEEE Transactions on Dielectrics and Electrical Insulation, 2011, Volume 18, Issue 5

[4] Wang, Xia; Lv, Zepeng; Wu, Kai; et al., "Study of the factors that suppress space charge accumulation in LDPE nanocomposites", IEEE Transactions on Dielectrics and Electrical Insulation, 2014, Volume 21, Issue 4

[5] Lau, K. Y; Vaughan, A. S; Chen, G; et al., "Space charge dynamics in silica-based polyethylene nanocomposites", 2013 IEEE International Conference on Solid Dielectrics (ICSD), 2013

[6] G. C. Montanari, "The electrical degradation threshold of polyethylene investigated by space charge and conduction current measurements", IEEE Trans. Dielectr. Electr. Insul., Vol. 7, N. 3, pp. 309-315, 2000.

[7] G.C. Montanari, "Notes on theoretical and practical aspects of polymeric insulation aging”, IEEE Electrical Insulation Magazine, Vol. 29, n. 4, pp. 30-40, August 2013.

[8] A. Cavallini, D. Fabiani, G. Mazzanti, G.C. Montanari, "Life model based on space-charge quantities for HVDC polymeric cables subjected to voltage-polarity inversions", IEEE Trans. on Dielectrics and Electrical Insulation, Vol. 9, n. 4, pp. 514-523, August 2002.

[9] G.C. Montanari, "Bringing an insulation to failure: the role of space charge", IEEE Trans. on Dielectrics and Electrical Insulation, Vol. 18, n. 2, pp. 339-364, April 2011.

[10] G. Mazzanti, G.C. Montanari, L. Dissado, "Electrical ageing and life models: the role of space charge", IEEE Trans. on Dielectrics and Electrical Insulation, Vol. 12, n. 5, pp. 876-890, October 2005.

[11] G. C. Montanari, "Relation between space charge and polymeric insulation ageing: cause and effect", IEE Proc. Science, Measurement and Technology, Vol. 150, pp. 53-57, 2003.

[12] G. C. Montanari, G. Mazzanti, F. Palmieri, A. Motori, G. Perego, S. Serra "Space-charge trapping and conduction in LDPE, HDPE and XLPE", J. Phys. D: Appl. Phys. 34 2902-2911, 2001.

[13] Fleming, R. J., Mogens Henriksen, and J. T. Holboll. "The influence of electrodes and conditioning on space charge accumulation in XLPE." IEEE transactions on dielectrics and electrical insulation 7.4 (2000): 561571.

[14] I. Rytöluoto, K. Lahti, M. Karttunen, and M. Koponen, "Large-area dielectric breakdown performance of polymer films - Part I: Measurement method evaluation and statistical considerations on area-dependence," IEEE Trans. Dielectr. Electr. Insul., vol. 22, no. 2, pp. 689-700, 2015.

[15] I. Rytöluoto, K. Lahti, M. Karttunen, M. Koponen, S. Virtanen, and M. Pettersson, "Large-area dielectric breakdown performance of polymer films - Part II: Interdependence of filler content, processing and breakdown performance in polypropylene-silica nanocomposites," IEEE Trans. Dielectr. Electr. Insul., vol. 22, no. 4, pp. 2196-2206, 2015. 